\title{
Depression and falls among community dwelling elderly people: a search for common risk factors
}

\author{
A Biderman, J Cwikel, A V Fried, D Galinsky
}

J Epidemiol Community Health 2002;56:631-636

\begin{abstract}
Study objectives: Depression and falls are two common conditions that impair the health of older people. Both are relatively underdiagnosed and undertreated problems in primary care. The study objective was to investigate whether there was a common set of risk factors that could predict an increased risk of both falls and depression.

Design: This was a cohort study drawn from a primary care clinic, with a one year follow up. Dependent measures included: reporting two or more falls in the past year and a score of 7 or over on the S-GDS (Short Geriatric Depression Scale). A parsimonious set of risk factors was selected that predicted both outcomes based on a series of discriminant function analyses.

Participants and setting: The setting was a primary care clinic serving a mixed socioeconomic population, in Beer Sheva, Israel. The sample included 283 General Sick Fund members, aged 60 and over, who completed both baseline assessments and one year follow up interviews. Main results: At the one year follow up, $12 \%$ of the sample reported frequent falls in the past year and $25.5 \%$ of the sample screened positive for depressive symptoms. A set of five risk factors that included: poor self rated health, poor cognitive status, impaired ADL, two or more clinic visits in the past month, and slow walking speed (>10 seconds over five metres) was successful at discriminating between fallers and non-fallers (86\% discrimination) and between those with and without depressive symptoms $(76 \%)$. For every risk factor added, there was a significant increase in the proportion of respondents who had depressive symptoms. A similar result was found for falls.

Conclusions: These results show that there is a common set of risk factors that increase the risk of two common outcomes in geriatric medicine, falls and depression. For a general practitioner or a geriatric physician, it might be easier to detect these risk factors than to diagnose depression or high risk for falls. When these risk factors are detected in patients the physician can then be more active in direct probing about depression and falls.
\end{abstract}

See end of article for authors' affiliations

Correspondence to:

Department of Family Medicine, Faculty of Medical Sciences, Ben Gurion University of the Negev, POB 653, Beer Sheva, Israel 84105 ; sbider@netvision.net.il

$\mathrm{F}$ alls and depression are common problems in the elderly that generally escape detection and therefore go untreated in primary care. ${ }^{12}$ There is a consensus, however, that their prevention and treatment could greatly benefit the health and wellbeing of the elderly population. ${ }^{3}$ The development of a clearer understanding of their causes would help to formulate more effective means of prevention and treatment.

The reported prevalence of mild depressive symptoms among community dwelling elderly people over the age of 65 is between $15 \%-20 \%{ }^{4}$ although prevalence of major depressive disorders is only $2 \%-5 \% .{ }^{4}$ The vast majority of elderly people, an estimated $80 \%$, who suffer from symptoms of depression will not receive any treatment. ${ }^{6}$

Falls are also a common problem among elderly people; approximately $30 \%$ of those over 65 will fall each year, with rates rising with increasing age reaching about $40 \%$ in those over age $80 .{ }^{7}$ While most falls do not result in fractures, the $5 \%$ of falls that do can lead to complications and even death.

Depression is associated with falls among elderly people, although the order of causation is not clear. Unravelling the causal order would pinpoint the timing at which intervention is critical and suggest more specific modes of treatment. There are at least three different ways in which depression and falls may be related in general practice. Depression may precede a fall, or vice versa or that both are outcomes of a third factor that adversely affects health in older persons, and they develop concurrently. There is evidence for all three possibilities.

Depression preceded falls in a study of 336 persons aged 75 and over followed up prospectively, which found that the relative risk of falls was 1.7 when comparing the rate of falls among depressed elderly people and those without

depression. ${ }^{7}$ Another prospective study found that depression was a risk factor for falls among older women. ${ }^{8}$ Falls often bring depressive reactions and fear of falling among community dwelling elderly people. ${ }^{9}$ Fear of falling is common among elderly persons who have fallen, and even among those who have not fallen recently. ${ }^{7}$ The combined evidence for depression as a result of falls is less compelling than the depressionfalls causal direction.

The third type of relation between depression and falls postulates that they are both the result of a third condition or set of factors. Both depression and falls are known to be cross sectionally related to the presence of chronic medical conditions such as functional disability, ${ }^{10}$ and to prospectively predict a decline in physical functioning. ${ }^{11}$ Tinetti and colleagues found that a high score on depression and anxiety was one of four predisposing factors for falls, incontinence, and functional dependence. ${ }^{11}$

The purpose of this study is to search for a common set of risk factors that predict falls and depression among community dwelling elderly persons. Specific aims included: (1) whether a parsimonious set of factors could be identified that successfully predicts depression and falls; (2) whether adding depression to the set of shared risk factors improved our ability to predict falls, and visa versa.

\section{METHODS}

\section{Subjects}

The subjects were members of the General Sick Fund (Kupat Holim), the largest Health Maintenance Organisation in Israel, which was associated with the Labor Federation. 
Respondents residing in the catchment area of an urban, community primary care clinic in Beer Sheva were asked to take part in a demonstration project. Of the 567 elderly persons who met the inclusion criteria (age over 60, independent function, ability to take part in an interview) 361 (64\%) signed an informed consent form according to Helsinki Committee approval and agreed to take part in a project on fall prevention (NEFI- Negev Elderly Fall Intervention). Reasons for non-participation included lack of time or interest, inconvenience, or disinclination to become involved in an intervention programme. The respondents were interviewed either in their homes or in the clinic by trained interviewers. Interviews were conducted in Hebrew, Russian, Romanian, Spanish, English, and French depending on the preference of the respondent. At a one year follow up, 283 (78\%) were re-interviewed. The main reasons for non-completion of second year interviews included inability to renew contact $(n=15)$, refusal $(n=15)$, death $(n=7)$, significant health problems $(n=7)$, moved out of town $(n=9)$, other reasons $(n=25)$. There were no significant differences between those who participated in the second interview and those who did not on falls, ADL, IADL, self rated health, cognitive impairment, and number of clinic visits $(p=0.90,0.85,0.19,0.50,0.78,0.43$, respectively). Among the seven persons who died, there was a significant trend toward high scores of the depression scale ( 5 of 7 scored in the depressed range) and those who were lost to contact were also more likely to have had higher depression scores than those who were re-interviewed (34.3\% versus $17.5 \%$, $\mathrm{p}=0.03$ ).

\section{Measures}

The baseline questionnaire included information on demographic variables, functional (ADL and IADL) status, self reported health assessment and change in health status, clinic visits, hospitalisations, chronic medical problems, and use of medications. Specific questions were asked about the frequency of meetings with friends and family. A series of 12 questions were asked about social and leisure time activities. Those who reported doing three or less were considered inactive. Both self rated health and self rated physical activity were based on single questions measured on a five point scale. The medical problems included heart disease, hypertension, diabetes, CVA, COPD, orthopaedic problems, muscular weakness, vision and hearing impairments, and mental illness. We defined vision and hearing problems as those that were not correctable by glasses or hearing aids. Respondents were asked whether they regularly used the following types of medications: diuretics, laxatives, digoxin, $\beta$ blockers, corticosteroids, tranquilizers and hypnotic medications, insulin, oral hypoglicaemics, anticoagulants, non-steroidal antiinflammatory drugs, and any other drugs. (The interviewers were instructed to write down the commercial name of the medications and then consulted with a project physician in order to code it correctly).

Falls were defined as "an event when you find yourself unintentionally on the floor after you were in a standing or sitting position". Retrospective falls in the past year were recorded, and those who had fallen were asked about the circumstances of the fall and its outcome. Only $0.3 \%$ of the sample in the baseline interview did not recall whether they had fallen or not and these were excluded from later analyses. Fallers were defined as having reported at least two falls in the past year. Depressive symptoms were evaluated using the short version of the Geriatric Depression Scale that has been validated for use in Israel. ${ }^{12}$ The depression scale was used with its validated cut off score ( 7 and above is considered to indicate depressive symptoms). Cognitive impairment was assessed using a shortened version of the Brookdale Cognitive Screening Test (BCST), which has also been validated in Israel. ${ }^{13}$ This test has six items, with each question being

\begin{tabular}{ll}
$\begin{array}{l}\text { Table } 1 \text { Characteristics of the sample at baseline } \\
(\mathrm{n}=361)\end{array}$ & \\
\hline & Number (\%) \\
\hline Age $(\mathrm{y})$ & $155(43)$ \\
$60-70$ & $191(53)$ \\
$71-80$ & $12(3)$ \\
$80+$ & $3(1)$ \\
Missing data & $71.52(4.4)$ \\
Mean (SD) age & $152(42)$ \\
Sex & $209(58)$ \\
Male & \\
Female & $200(55)$ \\
Country of origin & $107(30)$ \\
CIS* & $54(15)$ \\
Europe, America & $121(33)$ \\
North Africa, Asia & $131(36)$ \\
Year of immigration & $107(30)$ \\
Before 1975 & $2(1)$ \\
Between 1976-1988 & \\
After 1989 & $121(34)$ \\
Missing data & $183(51)$ \\
Education & $38(11)$ \\
<10 years & $19(4)$ \\
$10-12$ years & $9.6(3.4)$ \\
12+ & $244(67)$ \\
Missing data & $114(32)$ \\
Mean (SD) years of education & $3(1)$ \\
Family status & \\
Married & \\
Single, divorced, widowed & \\
Missing data & \\
\hline *Commonwealth of Independent States or the former Soviet Union. \\
\end{tabular}

scored for full, partial or no correct answer. The cut off score was more than two items completely wrong or four items partially wrong.

In addition, respondents took part in a fall-risk assessment procedure-EFST (Elderly Falls Screening Test)-that has been described in detail elsewhere. ${ }^{14}$ The EFST consists of five items, three questions about falls (using the same definition as mentioned above), injurious falls and near falls, measurement of walking speed (>10 seconds over five metres is considered a slow gait) and an assessment of unsteady or uneven gait (for example, shuffling feet, wide walking base). Interviewers were specially trained to conduct the EFST. The one year follow up interview included the same items that measured the outcomes of interest in this study; falls and depression.

\section{Statistical methods}

The descriptive variables that describe the population were analysed by univariate distributions. Then bivariate analyses identified significant risk factors at baseline that predicted both depression and falls in the follow up period. All variables were dichotomised at either validated cut off points (depression, cognitive status) or at points where a physician would consider this a significant indicator of poor health (for example, greater than three chronic conditions, three or more routine medications, any ADL limitation). Unadjusted relative risk ratios (RR) were calculated. It was striking to note how many of the risk factors were common to both depression and falls. Those risk factors that were significantly associated with both depression and falls in the follow up period were entered into a series of discriminant function analyses to select a parsimonious group of factors that were common to both outcomes. When a set of five common factors were found, an index was created that counted the number of risk factors. Falls in the past year and a high score on the depression scale were added to this index to determine its success in predicting depression and falls, respectively over the one year follow up. 
Table 2 Sociodemographic, health, and functional risk factors for falls and for depression among community living elderly assessed at one year follow up $(n=283)$

\begin{tabular}{|c|c|c|c|}
\hline \multirow[b]{2}{*}{ Factor } & \multirow[b]{2}{*}{ Prevalence \% } & \multicolumn{2}{|l|}{$\operatorname{RR}(95 \% \mathrm{Cl}) \dagger$} \\
\hline & & Falls $\ddagger$ & Depression§̧ \\
\hline \multicolumn{4}{|l|}{ Sociodemographic } \\
\hline Age $>75$ & 15.5 & $0.56(0.18$ to 1.77$)$ & $0.81(0.44$ to 1.51$)$ \\
\hline Female & 58.8 & $3.93(1.57 \text { to } 9.87)^{* *}$ & $1.67(1.06 \text { to } 2.62)^{*}$ \\
\hline Education $>10 y$ & 34.1 & 1.93 (0.99 to 3.77$)$ & $1.22(0.80$ to 1.88$)$ \\
\hline Friends - infrequent meetings & 34.3 & $1.09(0.56$ to 2.13$)$ & $1.78(1.20 \text { to } 2.65)^{* *}$ \\
\hline Family status - unmarried & 31.8 & $2.28(1.21 \text { to } 4.30)^{*}$ & $1.22(0.81$ to 1.85$)$ \\
\hline \multicolumn{4}{|l|}{ Health related } \\
\hline Poor and very poor health (self rated) & 23.7 & $4.82(1.19 \text { to } 19.6)^{*}$ & $4.0(1.68 \text { to } 9.52)^{* * *}$ \\
\hline $\begin{array}{l}\text { Change for the worse in health status last } 6 \text { months (self } \\
\text { rated) }\end{array}$ & 27.7 & 1.32 (0.67 to 2.59$)$ & 1.45 (0.96 to 2.19$)$ \\
\hline$\geqslant 2$ clinic visits per month & 30.1 & $1.76(1.17 \text { to } 2.64)^{*}$ & $2.58(1.74 \text { to } 3.82)^{* * * *}$ \\
\hline$\geqslant 3$ Chronic diseases & 62.0 & $2.27(1.02 \text { to } 5.05)^{*}$ & $2.04(1.24 \text { to } 3.37)^{*}$ \\
\hline$\geqslant 3$ Medications taken & 41.2 & $1.93(1.01 \text { to } 3.69)^{*}$ & 1.55 (1.04 to 2.32$)^{*}$ \\
\hline Hospitalisation - any, during last year & 23.3 & $1.56(0.75$ to 3.23$)$ & $1.69(1.10 \text { to } 2.60)^{*}$ \\
\hline Vision problems & 15.9 & 0.95 (0.39 to 2.32$)$ & 1.44 (0.89 to 2.34$)$ \\
\hline Hearing problems & 5.5 & 2.34 (0.95 to 5.80$)$ & 1.36 (0.64 to 2.87$)$ \\
\hline \multicolumn{4}{|l|}{ Physical and leisure activities } \\
\hline ADL (any limitations) & 10.6 & $6.23(3.51 \text { to } 11.04)^{* * * *}$ & $3.76(2.69 \text { to } 5.24)^{* * * *}$ \\
\hline IADL (2 or more limitations) & 34.7 & $5.89(2.76 \text { to } 12.54)^{* * * *}$ & $2.46(1.64 \text { to } 3.68)^{* * * *}$ \\
\hline Total leisure activities $<3$ & 11.3 & $0.75(0.58 \text { to } 0.96)^{* * *}$ & $1.91(1.21 \text { to } 3.01)^{*}$ \\
\hline Physical activity (self rated) & 35.4 & $2.19(1.16 \text { to } 4.14)^{*}$ & $1.50(1.0 \text { to } 2.25)^{*}$ \\
\hline \multicolumn{4}{|l|}{ Affective/cognitive } \\
\hline Depression at baseline & 16.8 & $2.83(1.50 \text { to } 5.34)^{* *}$ & $1.85(1.22 \text { to } 2.81)^{* *}$ \\
\hline Cognitive impairment & 7.8 & $2.27(0.97$ to 5.30$)$ & $1.99(1.19 \text { to } 3.31)^{*}$ \\
\hline \multicolumn{4}{|l|}{ Falls and gait } \\
\hline$>$ one fall past year & 11.2 & $6.64(3.75 \text { to } 11.74)^{* * *}$ & $2.58(1.74 \text { to } 3.82)^{* * * *}$ \\
\hline Near falls often or occasionally & 26.0 & $1.29(1.10 \text { to } 1.51)^{* * * *}$ & $1.57(1.23 \text { to } 1.99)^{* * * *}$ \\
\hline Slow walking speed & 20.0 & $1.41(1.16 \text { to } 1.73)^{* * * *}$ & $1.75(1.28 \text { to } 2.37)^{* * * *}$ \\
\hline Unsteady/uneven gait & 28.0 & $1.26(1.10 \text { to } 1.43)^{* * * *}$ & $1.64(1.31 \text { to } 2.04)^{* * * *}$ \\
\hline Injurious fall in the past year & 8.5 & $1.35(0.84$ to 2.16$)$ & $1.08(0.63$ to 1.87$)$ \\
\hline
\end{tabular}

We looked separately at new incident cases of both depression and falls to generate hypotheses but found that the numbers were too small to conduct separate multivariate analyses. Ideally, the detection of separate risk factors should focus on such incident cases in order to find unique predictors for falls and depression, but this was not possible with these data. There were only 17 new fallers among elderly persons who did not report falls at baseline and 48 persons with high scores on the depression scale who did not have high scores at baseline.

\section{RESULTS}

The main demographic features of the study population are shown in table 1 . This study concentrated on the young-old as reflected by the mean age of 71 . The sample did not include any native born Israelis; around half originated in the former Soviet Union (CIS), a third came from Europe or the Americas, and 15\% were from North Africa and Asia. A third of the sample were relative veterans in Israel (immigrated before 1975), a third came between 1976-1988, and a third came in the recent wave of immigration from CIS since 1989. The majority of respondents had a partial high school education, were married and living with their spouse. The proportion of women in the population and the proportion of elderly persons who were married and living with their spouse is representative of urban, Israeli elderly persons. There were proportionately more immigrant elderly persons in the sample than in most urban locations, but this is common to the city of Beer Sheva, which has a high percentage of new immigrants. ${ }^{15}$

The relation between the demographic, health status, physical activity, affective state, and fall history and the reporting of either falls or depression at the one year follow up is shown in table 2. Each factor is shown by the category representing a poorer state of health, and the risk ratio was calculated using the category that is indicative of better health as the index group. Among the sociodemographic variables, only female gender is a consistent risk factor for both falls and depression. Most of the health related variables were consistent risk factors for both falls and depression, except a reported change for the worse in health status and problems with hearing or vision. In an effort to find specific medications that were related to falls or depression, we examined each medication in a separate series of bivariate analyses. Of the 10 medications, sedatives and hypnotics were significantly related to depression $(\mathrm{p}=0.004)$ and non-steroidal antiinflammatory drugs (NSAIDs) were associated with falls $(p=0.03)$. Limitations in activities of daily living (both basic and instrumental) were strongly associated with both outcomes, as were most of the parameters of gait, balance, and fall history. Depression at baseline was a significant risk factor for both outcomes, while cognitive impairment was significant only for depression.

We arrived at a parsimonious set of combined risk factors through a series of discriminant function analyses. The factors that were retained included: poor self rated health, poor cognitive status, impaired ADL, two or more clinic visits in the past month, and slow walking speed. As both ADL and IADL are markers for functional disability, we retained only ADL. The balance and gait factors from the fall risk assessment also were closely related, and so walking speed was retained as it was the most significant single factor. Each factor that was retained remained significant in three quarters of the final discriminant functions. Depression at baseline was also retained in predicting falls, as were retrospective falls in the year before baseline in predicting depression. The discriminant functions were successful at distinguishing between fallers and non-fallers (82\%) and those with depressed symptoms from those without $(76 \%)$. The results were better at identifying the non-cases than the cases $(84 \%$ for non-fallers, $64 \%$ for fallers; $82 \%$ for not depressed, $59 \%$ for those with 


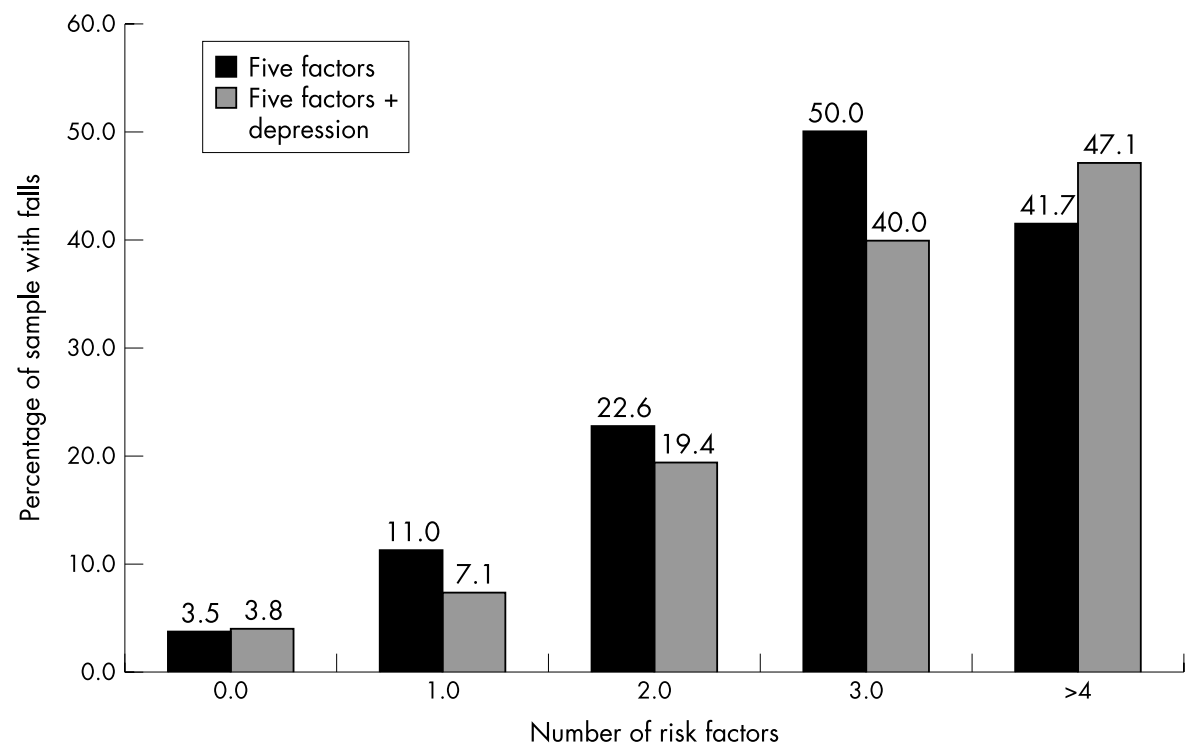

Figure 1 Occurrence of falls in the past year on the basis of the set of risk factors (poor self rated health, poor cognitive status, impaired ADL, two or more clinic visits in the past month, slow walking speed).

depressive symptoms). The discriminant analysis results did not change when depressive symptoms were added to the predictors for falls. However, the addition of falls to the set of predictors improved the case detection of depression from 59\% to $63 \%$.

Figure 1 shows the percentage of the sample that reported falls by the number of risk factors. The second column shows the model with the addition of depressive symptoms. There was no set order in which risk factors were added and therefore any additional factor increased the risk of falls. There was a significant increase in discriminating fallers from nonfallers with the addition of each factor up until three factors, at which point there is a non-significant decrease $\left(\chi^{2}\right.$ for trend $=28.2, p<0.00001)$. With the addition of depression, there is an increase in falls reported at each level $\left(\chi^{2}\right.$ for trend $=29.6, p<0.00001)$. However, the addition of depression as a risk factor did not improve the explanatory power $(\mathrm{p}=0.79)$.

As there were no significant changes between the level at three risk factors and the level at four or more, these were combined as is shown in figure 2. Here, it is clear that the set of risk factors succeeds very well in predicting both depressive symptoms and falls. The proportion of respondents reporting both falls and depressive symptoms increases dramatically for each risk factor added. In the case of depressive symptoms, the proportion of respondents increases from 14\% with no risk factors to $78 \%$ with three or more risk factors, yielding a relative risk of $6.6\left(\chi^{2}\right.$ for linear trend $\left.=29.7, p<0.0001\right)$. The addition of falls to the set of risk factors did not improve the predictive power for depression (similar to predicting falls in fig 1). With falls, the increase in the proportion of respondents who reported falls increased from $3.5 \%$ with no risk factors to $46 \%$ with three or more risk factors, yielding a relative risk of $13.4\left(\chi^{2}\right.$ for linear trend $\left.=29.0, p<0.0001\right)$.

\section{DISCUSSION}

The results of this study support the third hypothesis about the relation between falls and depression as common outcomes from a set of other risk factors. We were able to identify five risk factors that individually and in combination increased the risk of falls and depression among community dwelling elderly persons seen in primary care. The five risk factors identified were poor self rated health, poor cognitive function, impaired ADL, two or more clinic visits in the past month, and slow walking speed. They represent different, yet overlapping domains in geriatric assessment: medical conditions, psychosocial status, cognitive function, and functional status. Self rated health and two or more recent clinic visits can indicate both acute and chronic morbidity with the addition of negative effect on morale. ${ }^{16}$ Frequent clinic visits may also be a sign of help seeking, a need for attention, or an indication of a lack of an adequate social support. ${ }^{17}$ Poor cognitive function reflects neuropsychological status and may be associated with depression. ${ }^{18}$ Impaired ADL is an expression of functional dependence and one of the most commonly used measures in assessing health in old age. Slow walking speed

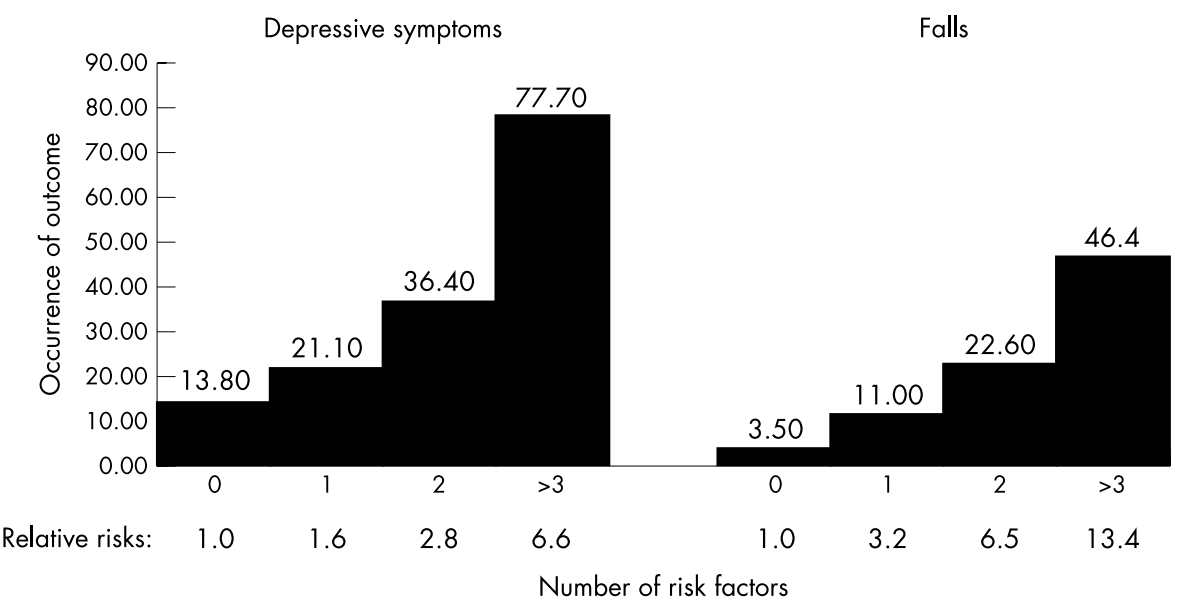

Figure 2 Occurrence of falls and depressive symptoms on the basis of the set of risk factors (poor self rated health, poor cognitive status, impaired ADL, two or more clinic visits in the past month, slow walking speed). 


\section{Key points}

- Depression and falls among elderly persons are two common conditions that are relatively underdiagnosed and undertreated in primary care.

- The objective was to investigate whether there was a common set of risk factors that could predict an increased risk of both falls and depression.

- The setting was a primary care clinic serving a mixed socioeconomic population, in Beer Sheva, Israel. The sample included 283 General Sick Fund members, aged 60 and over, who completed both baseline assessments and one year follow up interviews.

- At the one year follow up, $12 \%$ of the sample reported frequent falls in the past year and $25.5 \%$ of the sample screened positive for depressive symptoms.

- A set of five risk factors was successful at discriminating between fallers and non-fallers (86\% discrimination) and between those with and without depressive symptoms $(76 \%)$. The risk factors found were poor self rated health, poor cognitive status, impaired ADL, two or more clinic visits in the past month, and slow walking speed (>10 seconds over five metres)

- For every risk factor added, there was a significant increase in the proportion of respondents who had depressive symptoms. A similar result was found for falls.

- For a general practitioner or a geriatric physician, it might be easier to detect these risk factors than to diagnose depression or high risk for falls. When these risk factors are detected in patients the physician can then be more active in direct probing about depression and falls.

represents psychomotor performance that combines physical agility, strength and proprioception and may also be an expression of psychological status, especially depression. ${ }^{19}$ The findings are consistent with the principle of "multifinality", which means that the presence of a specific risk factor can lead to multiple outcomes, reinforcing the need to adopt a multifactorial model for the change in health status among the elderly.

The identification of depression among the elderly population has been shown to be problematic in primary care. ${ }^{1}$ Typically, primary care physicians only recognise a small portion (around 20\%) of elderly patients with depression. The main reason is that many elderly persons somatise their depression in physical symptoms. ${ }^{20}$ In a similar fashion, older people are often reluctant to report falls to their primary care physician, or they simply forget about their occurrence. ${ }^{21}$

It may be more feasible to identify the risk factors for depression and falls than the conditions themselves. The selected risk factors are all indicators that are easily assessed in primary care practice. Those who have several of these risk factors concurrently should be actively assessed for their psychological state and fall risk. Many of these issues become more clear after a home visit by the family physician or community nurse with environmental assessment. In a home visit, it is possible to assess home hazards for falls and changes in the psychosocial environment, such as a recent illness of a family member, family abuse or neglect, and bereavement.

In this study, we used low ADL as a risk factor for falls and depression. Clinical observations and the literature led us to believe that decline in ADL was more likely to precede the development of depression or falls. ${ }^{722}$ In other studies, ADL or physical disability have been used as outcome variables (for example, Tinetti $e t a l^{11}$ and Bruce $e t a^{24}$ ). As others have noted, any one condition can initiate a process of decline in health status in elderly persons, which may then involve other psychophysiological systems leading to functional impairment and disability. ${ }^{24}$

Leisure time physical activity and exercise have been shown to be positively associated with general wellbeing, and lower levels of depression and anxiety, while protecting from the risk of fracture and falls. ${ }^{25}{ }^{26}$ Our results on the centrality of walking speed and ADL are consistent with these findings. Exercise programmes especially designed for older persons are a promising area for fall prevention. ${ }^{26}$

The results in this study did not implicate hearing and visual problems as risk factors for falls despite the fact that these have been often reported. ${ }^{22}$ This may either be because of the relatively small number of respondents with either visual or hearing problems in the study, but probably because of the young-old age group and independent status of the study sample.

The high rate of participation in the study (64\%) indicates that the issues of mobility and falls are of interest to a broad selection of independently dwelling elderly persons. We have no further information on those who chose not to participate in the project at Time 1, but their reasons for declining indicate that they may be a more active group.

The study relied on self report of falls, which is known to have some systematic reporting bias because of retrospective recall. ${ }^{2}$ However, this practice is consistent with other research protocols that have found that reported falls are an adequate proxy measure for fall incidence. ${ }^{723}$ Furthermore, the other results in the study give strong convergent validity to the fall reports.

The multifactorial causation of falls and depression suggests that the most appropriate treatment approach is multidisciplinary, involving medical, functional, and psychosocial aspects of geriatric care. Identification of those patients at high risk of either depression or falls by attending to the accumulation of risk factors, can be a step in primary prevention. The physician's recommendation can not only improve compliance to medication, but can positively influence attendance at physical exercise and social activities and other lifestyle changes. In interviewing the elderly patient, it is worth asking specifically about the occurrence of falls and to use simple depression screening tests, for example. ${ }^{27}$

In conclusion, falls and depression are common and interrelated symptoms in the elderly. More studies are needed to further elucidate their relation and to strengthen efforts to prevent their occurrence or at least to detect and treat them effectively. This may afford a better quality of life for the rapidly growing elderly segment of the population.

\section{Authors' affiliations}

A Biderman, Department of Family Medicine, Faculty of Medical Sciences, Ben Gurion University of the Negev, and Klalit Health Services, Beer Sheva, Israel

J Cwikel, Department of Social Work and Centre for Women's Health Studies and Promotion, Ben Gurion University of the Negev

A V Fried, D Galinsky, Department of Geriatric Medicine, Soroka Medical Centre, Faculty of Medical Sciences, Ben Gurion University of the Negev

Funding: this work was partially supported by a grant from Israel's National Insurance Institute, Division on Special Projects and ESHELJOINT. The work was based on the NEFI (Negev Elderly Falls

Intervention) project.

Conflicts of interest: none.

\section{REFERENCES}

1 Banazak DA. Late life depression in primary care: how well are we doing? J Gen Intern Med 1996;1 1:163-7.

2 Cumming RG, Kelsey JL, Nevitt MC. Methodologic issues in the study of frequent health problems: falls in the elderly. Ann Epidemio 1990;1:49-56.

3 Ory MG, Cox DM. Forging ahead: linking health and behavior to improve quality of life in older people. Social Indicators Research 1994:33:89-120.

4 Blazer D, Williams CD. Epidemiology of dysphoric and depression in an elderly population. Am J Psychiatry 1980;137:439-44.

5 Macdonald AJD. Mental health in old age. BM 1997;315:413-17.

6 Perez-Stable EJ, Miranda J, Munoz RF, et al. Depression in medical outpatients. Underrecognition and misdiagnosis. Arch Intern Med 1990; 150:1083-8. 
7 Tinetti ME, Speechley M, Ginter SF. Risk factors for falls among elderly persons living in the community. N Engl J Med 1988;319:1701-7.

8 Whooley MA, Kip KE, Cauley JA, et al. Depression, falls and risk of fracture in older women. Study of Osteoporotic Fractures Research Group. Arch Intern Med 1999:159:484-90.

9 Arfken CL, Lach HW, Birge SJ, et al. The prevalence and correlates of fear of falling in elderly persons living in the community. Am J Public Health 1994; 84:565-70.

10 Berkman LF, Berkman CS, Karl S, et al. Depressive symptoms in relation to physical health and functioning in the elderly. Am J Epidemiol 1986;124:372-87.

11 Tinetti ME, Inouye SK, Gill TM, et al. Shared risk factors for falls, incontinence and functional dependence: unifying the approach to geriatric syndromes. JAMA 1995;273:1348-53.

12 Cwikel J, Ritchie K. Screening for depression among the elderly in Israel: an assessment of the Short Geriatric Depression Scale (S-GDS). Isr J Med Sci 1989;25:131-7.

13 Davies AM. Epidemiology of senile dementia in Jerusalem. Jerusalem: Brookdale Institute, 1988

14 Cwikel JG, Fried AV, Biderman A, et al. Validation of a fall-risk screening test (EFST) for community-dwelling elderly. Disabil Rehabil 1998;20:161-7.

15 Brodsky J, Schnoor Y, Be'er S. The elderly in Israel-The 1998 Statistical Abstract. Jerusalem: Eshel (The Association for the Planning and Development of Services for the Aged in Israel and JDC) Brookdale Institute of Gerontology and Human Development, 1998

16 Demers M. Frequent users of ambulatory health care in Quebec: the case of doctor-shoppers. Can Med Assoc J 1995;153:37-42.
17 Ben-Sira Z. Primary care practitioners likelihood to engage in a bio-psychosocial approach: an additional perspective on the doctor-patient relationship. Soc Sci Med 1990;31:565-76.

18 Ballard CG, Bannister C, Oyebode F. Depression in dementia sufferers. International Journal of Geriatric Psychiatry 1996;11:507-15.

19 Sloman L, Berridge M, Homatidis MA, et al. Gait patterns of depressed patients and normal subjects. Am J Psychiatry 1982;139:94-7.

20 Cole MG, Yaffe MY. Pathway to psychiatric care of the elderly with depression. International Journal of Geriatric Psychiatry 1996;11:157-61

21 Cummings SR, Nevitt MC, Kidd S. Forgetting falls. The limited accuracy of recall in the elderly. J Am Geriatr Soc 1988;36:613-16.

22 Cwikel J, Fried AV. The social epidemiology of falls among community-dwelling elderly: guidelines of prevention. Disabil Rehabil 1992:14:113-21.

23 Nevitt MC, Cummings SR, Kidd S, et al. Risk factors for recurrent nonsyncopal falls: A prospective study. JAMA 1989;261:2663-8.

24 Bruce ML, Seeman TE, Merrill SS, et al. The impact of depressive symptomatology on physical disability: MacArthur Studies of Successful Aging. Am J Public Health 1994:84:1796-9.

25 Stephens T. Physical activity and mental health in the United States and Canada: evidence from four population surveys. Prev Med $1988 ; 17: 35-47$

26 Province MA, Hadley EC, Hornbrook MC, et al. The effects of exercise on falls in elderly patients. A preplanned meta-analysis of the FICSIT Trials. JAMA 1995;273:1341-7.

27 Dorfman RA, Lubben JE, Mayer-Oakes A, et al. Screening for depression among a well elderly population. Social Work 1995;40:295-304 